CARPATHIAN J. MATH.

Volume 38 (2022), No. 2,

Pages 263 - 280

\title{
Viscosity algorithm for solving split generalized equilibrium problem and fixed-point problem
}

\author{
JAMILU ABUbAKAR ${ }^{1,2}$ and Jitsupa DeEPHO ${ }^{3^{*}}$
}

\begin{abstract}
This article considers a split generalized equilibrium problem and fixed point problem for infinite family of nonexpansive mapping in Hilbert space. We propose an algorithm for finding a common solution of these problems. Under mild assumptions, we establish a strong convergence theorem for the sequence generated by the proposed algorithm using viscosity technique. We present the implementation of the proposed algorithm by considering some numerical illustrations and comparison of the proposed algorithm with an existing algorithm in the literature.
\end{abstract}

\section{INTRODUCTION}

Throughout this article, we denote $C$ to be a nonempty closed and convex subset of a real Hilbert space $H_{1}$. A mapping $K$ of $C$ onto $H_{1}$ is said to be nonexpansive if for each $u, v \in C$

$$
\|K(u)-K(v)\| \leq\|u-v\| .
$$

We denote $F(K)$ to be the set of fixed points of $K$. It has been established that if $C$ is closed, bounded and convex, then $F(K)$ is nonempty (see for instance [19]). There are several methods for approximating the fixed points of $K$. For some recent numerical methods and application (Ref. $[1,9,10]$ ). Moudafi [11] introduced a viscosity iterative technique that starts with an arbitrary initial point $u_{0} \in C$, then defines recursively a sequence $\left\{u_{n}\right\}$ by

$$
u_{n+1}=\varrho_{n} l\left(u_{n}\right)+\left(1-\varrho_{n}\right) K u_{n} \forall n \geq 0,
$$

to approximate the fixed point of a nonexpansive mapping $K$, where $l$ is an $\alpha$-contraction on $C$ (that is, for each $u, v \in C,\|l(u)-l(v)\| \leq \alpha\|u-v\|$ with $\alpha \in(0,1)$ ). It is established in [11] that under standard conditions imposed on $\left\{\varrho_{n}\right\}$, the sequence $\left\{u_{n}\right\}$ generated by (1.1) strongly converges to the unique solution $u^{*} \in F i x(K)$, which is an optimal solution to the variational inequality problem (VIP)

$$
\left\langle(I-l) u^{*}, u-u^{*}\right\rangle \geq 0, \forall u \in F i x(K) .
$$

Suppose $F_{1}: C \times C \rightarrow \mathbb{R}$ with $F_{1}(u, u)=0$, for each $u \in C$ be a bifunction, an equilibrium problem (EP) involving the bifunction $F_{1}$ is a problem of finding a point $u \in$ $C$ such that

$$
F_{1}(u, v) \geq 0, \forall v \in C \text {. }
$$

In the sequel, we will denote the solution set of (1.3) by $E P\left(F_{1}\right)=\left\{u \in C: F_{1}(u, v) \geq 0\right.$, $\forall v \in C\}$. This problem contains as a special case many important problems, for instance, fixed point problems, optimization problems, variational inequality problem and Nash

Received: 24.03.2021. In revised form: 17.10.2021. Accepted: 08.12.2021

2010 Mathematics Subject Classification. 34K28, 47H09, 47H10, 47J40.

Key words and phrases. Split generalized equilibrium problem, Infinite nonexpansive mapping, Fixed point problem, Split equilibrium problem, Hilbert spaces.

Corresponding author: Jitsupa Deepho; jitsupa.d@sciee.kmutnb.ac.th 
equilibrium. Additionally, many problems arising in Engineering and Economics can be reduced to problem (1.3) (Ref. $[4,18]$ ).

Takahashi and Takahashi [18] introduced a viscosity approximation method for equilibriums and fixed point of a nonexpansive mapping in real Hilbert space based on the viscosity technique for nonexpansive mapping [11]. Under some suitable conditions, it is shown that the sequence generated by the method converges strongly to the solution of (1.3) and fixed point of a nonexpansive mapping.

Kazimi and Rizvi in [6] introduced the split equilibrium problem (SEP) for two nonlinear bifunctions $F_{1}: C \times C \rightarrow \mathbb{R}$ and $F_{2}: Q \times Q \rightarrow \mathbb{R}$, under a bounded linear transformation $A: H_{1} \rightarrow H_{2}$, where $Q$ is a closed and convex subset of a real Hilbert space $H_{2}$. The problem concerns with finding $u^{*} \in C$ such that

$$
F_{1}\left(u^{*}, u\right) \geq 0, \forall u \in C
$$

and such that

$$
v^{*}=A u^{*} \in Q \text { solves } F_{2}\left(v^{*}, v\right) \geq 0, \forall v \in Q .
$$

Observe that, the problems (1.4) and (1.5) consist of a pair of equilibrium problems and problem (1.4) separately, is exactly problem (1.3). For some recent iterative methods for solving $S E P$, the reader should refer to (Ref. $[16,24,8])$.

Recently, Kazmi and Rizvi in [7] further generalized SEP to a more general problem by incorporating two more additional nonlinear bifunctions $\psi_{1}: C \times C \rightarrow \mathbb{R}$ and $\psi_{2}$ : $Q \times Q \rightarrow \mathbb{R}$, to introduce a split generalized equilibrium problem (SGEP) as follows: Find $u^{*} \in C$ such that

$$
F_{1}\left(u^{*}, u\right)+\psi_{1}\left(u^{*}, u\right) \geq 0, \forall u \in C,
$$

and such that

$$
v^{*}=A u^{*} \in Q \text { solves } F_{2}\left(v^{*}, v\right)+\psi_{2}\left(v^{*}, v\right) \geq 0, \forall v \in Q .
$$

We denote the solution set of SGEP (1.6) and (1.7) by $\Gamma=\left\{u^{*} \in G E P\left(F_{1}, \psi_{1}\right): A u^{*} \in\right.$ $\left.E P\left(F_{2}, \psi_{2}\right)\right\}$. The authors in [7] proposed and studied an iterative method for solving the common solution of SGEP, variational inequality problem and fixed point of a nonexpansive mapping $K$. Given an initial point $u_{0} \in H_{1}$, the sequence $\left\{u_{n}\right\}_{n=1}^{\infty}$ is generated via

$$
\left\{\begin{array}{l}
v_{n}=T_{\mu_{n}}^{F_{1}}\left(u_{n}+\delta A^{*}\left(T_{\mu_{n}}^{F_{1}}-I\right) A u_{n}\right) \\
\omega_{n}=P_{C}\left(v_{n}-\eta_{n} N u_{n}\right) \\
u_{n+1}=\varrho_{n} \nu+\vartheta_{n} u_{n}+\theta_{n} K \omega_{n},
\end{array}\right.
$$

where $N: H_{1} \rightarrow H_{1}$ is a strongly monotone operator. Under some suitable conditions on the sequences $\mu_{n}, \eta_{n}, \varrho_{n}, \vartheta_{n}$ and $\theta_{n}$, the scheme (1.8) is shown to converge strongly to the common solution of the considered problem in Hilbert space.

Furthermore, Hay et. al. very recently in [5] proposed a general iterative method for approximations of common solution of split generalized equilibrium problems and fixed points of a finite family of nonexpansive mappings. The method is as follows: For a given $u_{1} \in C_{1}=C$ arbitrarily, for each $i=1,2, \cdots, N$ with positive integer $N$, the method generates the sequences $v_{n, i}, \omega_{n, i}$ and $u_{n}$ as follows

$$
\left\{\begin{array}{l}
v_{n, i}=T_{r_{n, i}}^{F_{i}, \psi_{i}}\left(u_{n}+\delta A_{i}^{*}\left(T_{r_{n, i}}^{F_{i}, \psi_{i}}-I\right) A_{i} u_{n}\right) \\
\omega_{n, i}=\eta_{n, i} u_{n} K_{i}+\left(1-\eta_{n, i}\right) v_{n, i} \\
C_{n+1, i}=\left\{u^{*} \in C_{n}:\left\|\omega_{n, i}-u^{*}\right\| \leq\left\|u_{n}-u^{*}\right\|\right\} \\
u_{n+1}=\zeta_{n} P_{C_{n+1}} u_{1}+\left(1-\zeta_{n}\right) \omega_{n, i} .
\end{array}\right.
$$


Under some appropriate conditions on the sequences $\eta_{n, i}, \zeta_{n}$ and $\delta$, strong convergence of the sequence generated by (1.9) is established. It can be observed that, the strong convergence of the sequences generated by (1.9) is obtained by the so called shrinking technique of which it requires the projection onto the closed and convex set $C_{n+1}$ for implementations. However, it is known that the projection onto a closed set is difficult and sometimes computationally expensive.

In this paper, based on the viscosity iteration method introduced in [11], motivated and inspired by the work of Kazmi and Rizvi [7], we introduce an iterative method that does not require any projection onto a closed set for finding a common solution of split generalized equilibrium problem and fixed point problem for infinite family of nonexpansive mappings in Hilbert spaces. Under some standard conditions, we show that the sequence generated by the proposed method strongly converges to the solution of the considered problem. In addition, we present some numerical illustrations to support our proposed strong convergence theorem. Moreover, we demonstrate the implementation and computational performance of the proposed method with some existing results in the literature.

We outlined the article as follows: In Section 2, we recalled some important definitions, Lemmas and results that are needed for the convergence analysis of the proposed method. In section 3, we present our main strong convergence theorem and its proof. In the last section, we report the numerical implementation.

\section{PRELiminARIES}

Let $C$ be a nonempty closed convex subset of a real Hilbert space $H_{1}$ with inner product and induced norm denoted respectively as $\langle\cdot, \cdot\rangle$ and $\|\cdot\|$. It is well known that for any $u_{1}, u_{2} \in H_{1}$ and $\kappa \in[0,1]$, we have

$$
\begin{gathered}
\left\|u_{1}-u_{2}\right\|^{2}=\left\|u_{1}\right\|^{2}-\left\|u_{2}\right\|^{2}-2\left\langle u_{1}-u_{2}, u_{2}\right\rangle, \\
\left\|u_{1}+u_{2}\right\|^{2} \leq\left\|u_{1}\right\|^{2}+2\left\langle u_{2}, u_{1}+u_{2}\right\rangle,
\end{gathered}
$$

and

$$
\left\|\kappa u_{1}+(1-\kappa) u_{2}\right\|^{2}=\kappa\left\|u_{1}\right\|^{2}+(1-\kappa)\left\|u_{2}\right\|^{2}-\kappa(1-\kappa)\left\|u_{1}-u_{2}\right\|^{2},
$$

We now state the standard assumptions on the bifunctions involved in the considered split generalized problem (1.6) and (1.7).

Assumption 2.1. Let $F_{1}: C \times C \rightarrow \mathbb{R}$ be a bifunction satisfying the following assumptions:

(1) $F_{1}\left(u_{1}, u_{1}\right) \geq 0, \forall u_{1} \in C$,

(2) $F_{1}$ is monotone, i.e. $F_{1}\left(u_{1}, u_{2}\right)+F_{1}\left(u_{2}, u_{1}\right) \leq 0, \forall u_{1}, u_{2} \in C$,

(3) $F_{1}$ is upper hemicontinuous, i.e., for each $u_{1}, u_{2}, u_{3} \in C, \limsup _{r \rightarrow 0} F_{1}\left(r u_{3}+(1-\right.$ $\left.r) u_{1}, u_{2}\right) \leq F_{1}\left(u_{1}, u_{2}\right)$

(4) For each $u_{1} \in C$ fixed, the function $u_{2} \rightarrow F_{1}\left(u_{1}, u_{2}\right)$ is convex and lower semicontinuous.

Furthermore, let $\psi_{1}: C \times C \rightarrow \mathbb{R}$ be such that

(1) $\psi_{1}\left(u_{1}, u_{1}\right) \geq 0, \forall u_{1} \in C$,

(2) For each $u_{2} \in C$ fixed, the function $u_{1} \rightarrow \psi_{1}\left(u_{1}, u_{2}\right)$ is upper hemicontinuous,

(3) For each $u_{1} \in C$ fixed, the function $u_{2} \rightarrow \psi_{1}\left(u_{1}, u_{2}\right)$ is convex and lower semicontinuous.

We recall the following result on the existence and some characterizations of proximity operator associated with the bifunctions for the generalized problem (1.6) and (1.7).

Lemma 2.1. [7] Suppose that the bifunctions $F_{1}, \psi_{1}: C \times C \rightarrow \mathbb{R}$ satisfy Assumption 2.1. Then for a positive number $\mu$ and $u_{3} \in H_{1}$, there exists $u_{1} \in C$ such that $F_{1}\left(u_{1}, u_{2}\right)+\psi_{1}\left(u_{1}, u_{2}\right)+$ $\frac{1}{\mu}\left\langle u_{2}-u_{1}, u_{1}-u_{3}\right\rangle \geq 0, \forall u_{2} \in C$. 
Lemma 2.2. [2] Suppose that the bifunctions $F_{1}, \psi_{1}: C \times C \rightarrow \mathbb{R}$ satisfy Assumption 2.1, $\psi_{1}$ is monotone and for any $u_{1} \in H_{1}$ and $\mu>0$, define a mapping $T_{\mu}^{\left(F_{1}, \psi_{1}\right)}: H_{1} \rightarrow C$ as follows:

$$
T_{\mu}^{\left(F_{1}, \psi_{1}\right)} u_{1}=\left\{u_{3} \in C: F_{1}\left(u_{1}, u_{2}\right)+\psi_{1}\left(u_{1}, u_{2}\right)+\frac{1}{\mu}\left\langle u_{2}-u_{1}, u_{1}-u_{3}\right\rangle \geq 0\right\}, \forall u_{2} \in C
$$

Then, the followings are true:

(1) $T_{\mu}^{\left(F_{1}, \psi_{1}\right)}$ is single - valued.

(2) For every $u_{1}, u_{2} \in H_{1}$

$$
\left\|T_{\mu}^{\left(F_{1}, \psi_{1}\right)} u_{1}-T_{\mu}^{\left(F_{1}, \psi_{1}\right)} u_{2}\right\|^{2} \leq\left\langle T_{\mu}^{\left(F_{1}, \psi_{1}\right)} u_{1}-T_{\mu}^{\left(F_{1}, \psi_{1}\right)} u_{2}, u_{1}-u_{2}\right\rangle,
$$

that is, $T_{\mu}^{\left(F_{1}, \psi_{1}\right)}$ is firmly nonexpansive.

(3) The fixed points set of Fix $\left(T_{\rho}^{\left(F_{1}, \psi_{1}\right)}\right)=\operatorname{GEP}\left(F_{1}, \psi_{1}\right)$.

(4) The solution set $\operatorname{GEP}\left(F_{1}, \psi_{1}\right)$ is convex and compact.

Similarly, if we suppose that $F_{2}, \psi_{2}: Q \times Q \rightarrow \mathbb{R}$ are bifunctions satisfying Assumption 2.1. Then for each $\rho>0$ and $v_{1} \in H_{2}$, a mapping $T_{\rho}^{\left(F_{2}, \psi_{2}\right)}: H_{2} \rightarrow Q$ is defined as:

$$
T_{\rho}^{\left(F_{2}, \psi_{2}\right)} v_{1}=\left\{v_{3} \in Q: F_{2}\left(v_{1}, v_{2}\right)+\psi_{2}\left(v_{1}, v_{2}\right)+\frac{1}{\rho}\left\langle v_{2}-v_{1}, v_{1}-v_{3}\right\rangle \geq 0\right\}, \forall v_{2} \in Q .
$$

Hence, it readily follows that, the mapping $T_{\rho}^{\left(F_{2}, \psi_{2}\right)}$ satisfies the characterizations (1), (2) in Lemma 2.2 and the solution set $\operatorname{GEP}\left(F_{2}, \psi_{2}\right)$ is also convex and compact.

Lemma 2.3. [3] Let $F_{1}: C \times C \rightarrow \mathbb{R}$ be a bifunction satisfying Assumption 2.1 and let the mapping $T_{\mu}^{F_{1}}$ be defined as in Lemma 2.2. Then, for every $u, v \in H_{1}$ and $\mu, \rho>0$, we have

$$
\left\|T_{\rho}^{F_{2}} v-T_{\mu}^{F_{1}} u\right\| \leq\|u-v\|+\left|\frac{\rho-\mu}{\rho}\right|\left\|T_{\rho}^{F_{2}} v-v\right\| .
$$

Lemma 2.4. [20] Let $F_{1}: C \times C \rightarrow \mathbb{R}$ be a bifunction satisfying Assumption 2.1 and for $\mu>0$ let $T_{\mu}^{F_{1}}$ be defined as in Lemma 2.2. Then for any $u \in H_{1}$ and $\rho>0$

$$
\left\|T_{\rho}^{F_{1}} u-T_{\mu}^{F_{1}} u\right\|^{2} \leq \frac{\rho-\mu}{\rho}\left\langle T_{\rho}^{F_{1}} u-T_{\mu}^{F_{1}} u, T_{\rho}^{F_{1}} u-u\right\rangle .
$$

The following Lemmas are essential for the convergence of the iterates generated by the proposed iterative method.

Lemma 2.5. [17] Let $\left\{u_{n}\right\}$ and $\left\{v_{n}\right\}$ be bounded sequences in a Banach space $\mathcal{X}$ and $\left\{\sigma_{n}\right\}$ be $a$ sequence in $[0,1]$ satisfying $0<\liminf _{n \rightarrow \infty} \sigma_{n} \leq \limsup _{n \rightarrow \infty} \sigma_{n}<1$. For all integers $n \geq 0$, let $u_{n+1}=\left(1-\sigma_{n}\right) v_{n}+\sigma_{n} u_{n}$ and $\lim _{\sup _{n \rightarrow \infty}}\left(\left\|v_{n+1}-v_{n}\right\|-\left\|u_{n+1}-u_{n}\right\|\right) \leq 0$. Then, $\lim _{n \rightarrow \infty}\left\|v_{n}-u_{n}\right\|=0$.

Lemma 2.6. [22] Suppose that $\left\{\zeta_{n}\right\}$ is a sequence of nonnegative real numbers satisfying $\zeta_{n+1} \leq$ $\left(1-\varrho_{n}\right) \zeta_{n}+\kappa_{n}, n \geq 0$ where $\left\{\varrho_{n}\right\}$ is a sequence in $(0,1)$ and $\left\{\kappa_{n}\right\}$ is a sequence in $\mathbb{R}$ such that (i) $\sum_{n=1}^{\infty} \varrho_{n}=\infty$ (ii) $\lim \sup _{n \rightarrow \infty} \frac{\kappa_{n}}{\varrho_{n}} \leq 0$ or (iii) $\sum_{n=1}^{\infty} \varrho_{n}<\infty$. Then $\lim _{n \rightarrow \infty} \zeta_{n}=0$.

Next, we introduce a mapping generated by infinite self-mappings of a closed and convex subset $C$ of $H_{1}$. For more on this mapping see [21]. Let $\sigma_{1}, \sigma_{2}, \ldots$ be real numbers such that $0 \leq \sigma_{i} \leq 1$ and $K_{1}, K_{2}, \ldots, K_{i}, \ldots$, be infinite mappings of $C$ into $C$ for each 
$i \in N$. For any $n \in N$, a mapping $W_{n}$ of $C$ into $C$ is defined recursively as follows:

$$
\begin{aligned}
U_{n, n+1} & =I, \\
U_{n, n} & =\sigma_{n} K_{n} U_{n, n+1}+\left(1-\sigma_{n}\right) I, \\
U_{n, n-1} & =\sigma_{n-1} K_{n-1} U_{n, n}+\left(1-\sigma_{n-1}\right) I, \\
& \vdots \\
U_{n, i} & =\sigma_{i} K_{i} U_{n, i+1}+\left(1-\sigma_{k}\right) I, \\
& \vdots \\
U_{n, 2} & =\sigma_{2} K_{2} U_{n, 3}+\left(1-\sigma_{2}\right) I, \\
W_{n}=U_{n, 1} & =\sigma_{1} K_{1} U_{n, 2}+\left(1-\sigma_{1}\right) I .
\end{aligned}
$$

is called the $W$-mapping generated by $K_{n}, K_{n-1}, \ldots, K_{1}$ and $\sigma_{n}, \sigma_{n-1}, \ldots, \sigma_{1}$.

Lemma 2.7. Let $C$ be a nonempty closed convex subset of a real Hilbert space $H_{1}$ and $K_{1}, K_{2}, \ldots$ be nonexpansive mappings of $C$ into $C$ such that $\cap_{i=1}^{\infty} F i x\left(K_{i}\right)$ is nonempty. Let $\sigma_{1}, \sigma_{2}, \ldots$ be real numbers such that for each $i \in N, 0<\sigma_{i} \leq b<1$. Then, for every $u \in C$, the $\lim _{n \rightarrow \infty} U_{n, i} u$ exists for each $i \in N$.

Remark 2.1. It is clear that, if $C$ is bounded, then from Lemma 2.7, for any $\varepsilon>0$ and $u \in C$, there exists a common positive integer number $N^{*}$ such that, $\left\|U_{n, i} u-U_{i} u\right\|<\varepsilon$ for all $n>N^{*}$. In fact, let $u^{*} \in \cap_{n=1}^{\infty} F i x\left(K_{n}\right)$, since $C$ is bounded, it can be seen by similar argument to Lemma 3.2 in [15], there exists a constant $N>0$ such that for all $u \in C,\left\|u-u^{*}\right\| \leq N$. Fix $i \in N$, then for any $u \in C$ and $n \in N$ with $n \geq i$, we have

$$
\left\|U_{n+1, k} u-U_{n, i} u\right\| \leq 2\left(\Pi_{k=i}^{n+1} \sigma_{k}\right)\left\|u-u^{*}\right\| \leq 2 N\left(\Pi_{k=i}^{n+1} \sigma_{k}\right) .
$$

Therefore, for $\varepsilon>0$, there exists $n^{*} \in N$ with $n^{*} \geq i$ such that for all $u \in C, b^{n^{*}-i+2}<$ $\varepsilon(1-b) / 2 N$. Thus, for every $m, n$ with $m>n>n^{*}$, we have

$$
\begin{aligned}
& \left\|U_{m, i} u-U_{n, i} u\right\| \\
\leq & \sum_{j=n}^{m-1}\left\|U_{j+1, i} u-U_{j, i} u\right\| \leq \sum_{j=n}^{m-1}\left\{2\left(\Pi_{j=k}^{j+1} \sigma_{k}\right)\left\|u-u^{*}\right\|\right\} \\
\leq & 2 N \sum_{j=n}^{m-1} b^{j-i+2} \leq \frac{2 N b^{n-i+2}}{1-b}<\varepsilon .
\end{aligned}
$$

Again, using Lemma 2.7, one can define a mapping $W$ of $C$ as $W u=\lim _{n \rightarrow \infty} W_{n} u=$ $\lim _{n \rightarrow \infty} U_{n, 1} u$. Such a $W$ is called the $W$-mapping generated by $K_{1}, K_{2}, \ldots$ and $\sigma_{1}, \sigma_{2}, \ldots$ We observe that if $\left\{u_{n}\right\}$ is a bounded sequence in $C$, then we have

$$
\lim _{n \rightarrow \infty}\left\|W u_{n}-W_{n} u_{n}\right\|=0 .
$$

In fact, from Remark 2.1, we have that, for any given $\varepsilon>0$, there exists $n^{*}$ such that $\| W u-$ $W_{n} u \| \leq \varepsilon$ for all $n \geq n^{*}$. In particular, $\left\|W u_{n}-W_{n} u_{n}\right\| \leq \varepsilon$ for all $n \geq n^{*}$. Consequently, $\lim _{n \rightarrow \infty}\left\|W u_{n}-W_{n} u_{n}\right\|=0$, as claimed. Throughout this article, we suppose that, for every $i \in N, 0<\sigma_{i} \leq b<1$.

Lemma 2.8. Let $C$ be a nonempty closed convex subset of a real Hilbert space $H$. Let $K_{1}, K_{2}, \ldots$ be nonexpansive mappings of $C$ into $C$ such that $\cap_{i=1}^{\infty} F i x\left(K_{i}\right)$ is nonempty, and let $\sigma_{1}, \sigma_{2}, \ldots$ be real numbers such that $0<\sigma_{i} \leq b<1$ for any $i \in N$. Then $F i x(W)=\cap_{i=1}^{\infty} F i x\left(K_{i}\right)$. 


\section{MAIN RESUlTS}

In this section, we present our proposed algorithm and the strong convergence result. Let $C \subset H_{1}, Q \subset H_{2}$ be nonempty closed and convex subsets of real Hilbert spaces $H_{1}$ and $H_{2}$ respectively. Let $F_{1}, \psi_{1}: C \times C \rightarrow \mathbb{R}$ and $F_{2}, \psi_{2}: Q \times Q \rightarrow \mathbb{R}$ be nonlinear bifunctions satisfying Assumption 2.1 with $F_{2}$ as upper semicontinuous in the first argument and for each $i \geq 1$, let $\left\{K_{i}\right\}_{i=1}^{\infty}$ be an infinite family of nonexpansive mappings of $C$ into $C$ such that $\cap_{i=1}^{\infty} F i x\left(K_{i}\right) \cap \Gamma \neq \emptyset$. Furthermore, let $f: H_{1} \rightarrow H_{1}$ be a contraction mapping with constant $\kappa \in(0,1)$ and $A: H_{1} \rightarrow H_{2}$ be a bounded linear operator with Adjoint operator $A^{*}$. Suppose that $\left\{\varrho_{n}\right\},\left\{\vartheta_{n}\right\}$ and $\left\{\theta_{n}\right\}$ are three sequences in $(0,1)$ such that $\varrho_{n}+\vartheta_{n}+\theta_{n}=1$ and the following conditions are satisfied.

(C1) $\lim _{n \rightarrow \infty} \varrho_{n}=0$ and $\sum_{n=1}^{\infty} \varrho_{n}=\infty$;

(C2) $0<\liminf _{n \rightarrow \infty} \vartheta_{n} \leq \limsup _{n \rightarrow \infty} \vartheta_{n}<1$;

(C3) $\liminf _{n \rightarrow \infty} \mu_{n}>0, \sum_{n=0}^{\infty}\left|\mu_{n+1}-\mu_{n}\right|<\infty$ and $\sum_{n=0}^{\infty}\left|\rho_{n+1}-\rho_{n}\right|=0$.

Algorithm 1: Viscosity-type Algorithm for GSEP

Initialization: Choose the sequence $\left\{\varrho_{n}\right\},\left\{\theta_{n}\right\},\left\{\vartheta_{n}\right\}$ in $(0,1)$ such that C1-C2 are satisfied and $\delta \in\left(0, \frac{1}{L^{2}}\right), L$ is the spectral radius of the operator $A^{*} A$. Given $\left\{\mu_{n}\right\} \subset[\mu, \infty)$ with $\mu>0,\left\{\rho_{n}\right\} \subset[\rho, \infty)$ with $\rho>0$ such that C3 is satisfied.

Iterative Steps: For $u_{n} \in H_{1}$, Compute $v_{n}$ as:

$$
v_{n}=T_{\rho_{n}}^{\left(F_{1}, \psi_{1}\right)}\left(u_{n}+\delta A^{*}\left(T_{\rho_{n}}^{\left(F_{2}, \psi_{2}\right)}-I\right) A u_{n}\right),
$$

Step 2. Compute

$$
u_{n+1}=\varrho_{n} f\left(u_{n}\right)+\vartheta_{n} u_{n}+\theta_{n} W_{n} v_{n},
$$

If $v_{n}=u_{n}$, stop $v_{n}$ is the solution of the SGEP, otherwise set $n:=n+1$ and go back to Step 1.

We are now ready to present our main strong convergence result.

Theorem 3.2. Let $H_{1}$ and $H_{2}$ be two real Hilbert spaces and $C \subset H_{1}, Q \subset H_{2}$ be nonempty closed and convex subsets. Let $F_{1}, \psi_{1}: C \times C \rightarrow \mathbb{R}$ and $F_{2}, \psi_{2}: Q \times Q \rightarrow \mathbb{R}$ be nonlinear mapping satisfying Assumption 2.1 and $F_{2}$ is upper semicontinuous in the first argument and for each $i \geq 1$, let $\left\{K_{i}\right\}_{i=1}^{\infty}$ be an infinite family of nonexpansive mappings of $C$ into $C$ such that $\cap_{i=1}^{\infty} F i x\left(K_{i}\right) \cap \Gamma \neq \emptyset$. Also, let $f: H_{1} \rightarrow H_{1}$ be a contraction mapping with constant $\kappa \in(0,1)$ and $A: H_{1} \rightarrow H_{2}$ be a bounded linear operator. Suppose that $\left\{\varrho_{n}\right\},\left\{\vartheta_{n}\right\}$ and $\left\{\theta_{n}\right\}$ are three sequences in $(0,1)$ such that C1-C2 are satisfied and $\varrho_{n}+\vartheta_{n}+\theta_{n}=1$. Given $u_{0} \in C$ arbitrary, the sequence $\left\{u_{n}\right\}$ generated by Algorithm 1 converges strongly to $u^{*}=P_{\cap_{i=1}^{\infty} F i x\left(K_{i}\right) \cap \Gamma} f\left(u^{*}\right)$.

Proof. For $\delta \in\left(0, \frac{1}{2 L^{2}}\right)$, the mapping $I+\delta A^{*}\left(T_{\rho_{n}}^{\left(F_{2}, \psi_{2}\right)}-I\right) A$ is a nonexpansive mapping and $A^{*}\left(T_{\rho_{n}}^{\left(F_{2}, \psi_{2}\right)}-I\right) A$ is a $\frac{1}{2 L^{2}}$ - inverse strongly monotone mapping. In fact, since $T_{\rho_{n}}^{\left(F_{2}, \psi_{2}\right)}$ 
is (firmly) nonexpansive and $T_{\rho_{n}}^{\left(F_{2}, \psi_{2}\right)}-I$ is $\frac{1}{2}$-inverse strongly monotone, we have

$$
\begin{aligned}
& \left\|A^{*}\left(T_{\rho_{n}}^{\left(F_{2}, \psi_{2}\right)}-I\right) A u-A^{*}\left(T_{\rho_{n}}^{\left(F_{2}, \psi_{2}\right)}-I\right) A v\right\|^{2} \\
= & \left\langle A^{*}\left(T_{\rho_{n}}^{\left(F_{2}, \psi_{2}\right)}-I\right)(A u-A v), A^{*}\left(T_{\rho_{n}}^{\left(F_{2}, \psi_{2}\right)}-I\right)(A u-A v)\right\rangle \\
= & \left\langle\left(T_{\rho_{n}}^{\left(F_{2}, \psi_{2}\right)}-I\right)(A u-A v), A A^{*}\left(T_{\rho_{n}}^{\left(F_{2}, \psi_{2}\right)}-I\right)(A u-A v)\right\rangle \\
\leq & L^{2}\left\langle\left(T_{\rho_{n}}^{\left(F_{2}, \psi_{2}\right)}-I\right)(A u-A v),\left(T_{\rho_{n}}^{\left(F_{2}, \psi_{2}\right)}-I\right)(A u-A v)\right\rangle \\
= & L^{2}\left\|\left(T_{\rho_{n}}^{\left(F_{2}, \psi_{2}\right)}-I\right)(A u-A v)\right\|^{2} \\
\leq & 2 L^{2}\left\langle A u-A v,\left(T_{\rho_{n}}^{\left(F_{2}, \psi_{2}\right)}-I\right)(A u-A v)\right\rangle \\
= & 2 L^{2}\left\langle u-v, A^{*}\left(T_{\rho_{n}}^{\left(F_{2}, \psi_{2}\right)}-I\right)(A u-A v)\right\rangle,
\end{aligned}
$$

for all $u, v \in H_{1}$, which implies that $A^{*}\left(T_{\rho_{n}}^{\left(F_{2}, \psi_{2}\right)}-I\right) A$ is a $\frac{1}{2 L^{2}}$ - inverse strongly monotone mapping. Note that $\delta \in\left(0, \frac{1}{L^{2}}\right)$. Thus $I+\delta A^{*}\left(T_{\rho_{n}}^{\left(F_{2}, \psi_{2}\right)}-I\right) A$ is nonexpansive mapping.

Step 1. We will show that, the sequence $\left\{u_{n}\right\}$ generated by Algorithm 1 is bounded.

Let $p \in \cap_{i=1}^{\infty} F i x\left(K_{i}\right) \cap \Gamma$ and $Q=P_{\cap_{i=1}^{\infty} F i x\left(K_{i}\right) \cap \Gamma} f(p)$. Note that, since $f$ is a contraction mapping with coefficient $\kappa \in(0,1)$, then

$$
\|Q f(u)-Q f(v)\| \leq\|f(u)-f(v)\| \leq \kappa\|u-v\|, \forall u, v \in H_{1} .
$$

Therefore $Q f$ is a contraction of $H_{1}$ into itself, which implies that there exists a unique element $p \in H_{1}$ such that $p=Q f(p)$. At the same time, we note that $p \in C$. Following a similar argument as in Theorem 3.1 in [23], one obtains

$$
\left\|v_{n}-p\right\|^{2} \leq\left\|u_{n}-p\right\|^{2}+\delta\left(\delta-\frac{1}{L^{2}}\right)\left\|A^{*}\left(T_{\rho_{n}}^{\left(F_{2}, \psi_{2}\right)}-I\right) A u_{n}\right\|^{2} .
$$

Since $\delta \in\left(0, \frac{1}{2 L^{2}}\right)$, we have

$$
\left\|v_{n}-p\right\| \leq\left\|u_{n}-p\right\|
$$

Therefore

$$
\begin{aligned}
\left\|u_{n+1}-p\right\| & \leq \varrho_{n}\left\|f\left(u_{n}\right)-p\right\|+\vartheta_{n}\left\|u_{n}-p\right\|+\theta_{n}\left\|W_{n} v_{n}-p\right\| \\
& \leq \varrho_{n}\left(\left\|f\left(u_{n}\right)-f(p)\right\|+\|f(p)-p\|\right)+\vartheta_{n}\left\|u_{n}-p\right\|+\theta_{n}\left\|v_{n}-p\right\| \\
& \leq \varrho_{n}\left(\kappa\left\|u_{n}-p\right\|+\|f(p)-p\|\right)+\left(1-\varrho_{n}\right)\left\|u_{n}-p\right\| \\
& \leq\left\{\kappa\left\|u_{0}-p\right\|, \frac{1}{1-\kappa}\|f(p)-p\|\right\} .
\end{aligned}
$$

Therefore, $\left\{u_{n}\right\}$ is bounded. Consequently, we have $\left\{v_{n}\right\},\left\{W_{n} u_{n}\right\}$ and $\left\{f\left(u_{n}\right)\right\}$ are all bounded.

Step 2. We will show that $\lim _{n \rightarrow \infty}\left\|u_{n+1}-u_{n}\right\|=0$ and $\lim _{n \rightarrow \infty}\left\|A^{*}\left(T_{\rho_{n}}^{\left(F_{2}, \psi_{2}\right)}-I\right) A u_{n}\right\|=0$.

Again by following similar arguments as in Theorem 3.1 in [23], we have

$$
\left\|v_{n+1}-v_{n}\right\| \leq\left\|u_{n+1}-u_{n}\right\|+\delta\|A\|\left(\frac{\left|\rho_{n+1}-\rho_{n}\right|}{\rho_{n+1}} \Omega_{n}\right)^{\frac{1}{2}}+\frac{\left|\mu_{n+1}-\mu_{n}\right|}{\mu_{n+1}} \Psi_{n+1},
$$

where

$\Psi_{n+1}=\sup _{n \in \mathbb{N}}\left\|T_{\mu_{n+1}}^{\left(F_{1}, \psi_{1}\right)}\left(u_{n+1}+\delta A^{*}\left(T_{\rho_{n+1}}^{\left(F_{2}, \psi_{2}\right)}-I\right) A u_{n+1}\right)-\left(u_{n+1}+\delta A^{*}\left(T_{\rho_{n+1}}^{\left(F_{2}, \psi_{2}\right)}-I\right) A u_{n+1}\right)\right\|$

and

$$
\Omega_{n}=\sup _{n \in \mathbb{N}}\left\langle T_{\rho_{n+1}}^{\left(F_{2}, \psi_{2}\right)} A u_{n}-T_{\rho_{n}}^{\left(F_{2}, \psi_{2}\right)} A u_{n}, T_{\rho_{n+1}}^{\left(F_{2}, \psi_{2}\right)} A u_{n}-A u_{n}\right\rangle
$$


Setting $u_{n+1}=\vartheta_{n} u_{n}+\left(1-\vartheta_{n}\right) z_{n}$ for all $n \geq 0$, we have that

$$
\begin{aligned}
z_{n+1}-z_{n}= & \frac{u_{n+2}-\vartheta_{n+1} u_{n+1}}{1-\vartheta_{n+1}}-\frac{u_{n+1}-\vartheta_{n} u_{n}}{1-\vartheta_{n}} \\
= & \frac{\varrho_{n+1}}{1-\vartheta_{n+1}}\left(f\left(u_{n+1}\right)-f\left(u_{n}\right)\right)+\left(\frac{\varrho_{n+1}}{1-\vartheta_{n+1}}-\frac{\varrho_{n}}{1-\vartheta_{n}}\right) f\left(u_{n}\right) \\
& +\frac{\theta_{n+1}}{1-\vartheta_{n+1}}\left(W_{n+1} v_{n+1}-W_{n} v_{n}\right)+\left(\frac{\theta_{n+1}}{1-\vartheta_{n+1}}-\frac{\theta_{n}}{1-\vartheta_{n}}\right) W_{n} v_{n} .
\end{aligned}
$$

Therefore, we have

$$
\begin{aligned}
\left\|z_{n+1}-z_{n}\right\| \leq & \frac{\kappa \varrho_{n+1}}{1-\vartheta_{n+1}}\left\|u_{n+1}-u_{n}\right\| \\
& +\left|\frac{\varrho_{n+1}}{1-\vartheta_{n+1}}-\frac{\varrho_{n}}{1-\vartheta_{n}}\right|\left(\left\|f\left(u_{n}\right)\right\|+\left\|W_{n} v_{n}\right\|\right) \\
& +\frac{\theta_{n+1}}{1-\vartheta_{n+1}}\left\|W_{n+1} v_{n+1}-W_{n} v_{n}\right\| .
\end{aligned}
$$

Since $K_{i}$ and $U_{n, i}$ are nonexpansive, then from (2.13), we have

$$
\begin{aligned}
\left\|W_{n+1} v_{n}-W_{n} v_{n}\right\| & =\left\|\sigma_{1} K_{1} U_{n+1,2} v_{n}-\sigma_{1} K_{1} U_{n, 2} v_{n}\right\| \\
& \leq \sigma_{1}\left\|U_{n+1,2} v_{n}-U_{n, 2} v_{n}\right\| \\
& \leq \sigma_{1} \sigma_{2}\left\|U_{n+1,3} v_{n}-U_{n, 3} v_{n}\right\| \\
& \leq \cdots \\
& \leq \sigma_{1} \sigma_{2} \ldots \sigma_{n}\left\|U_{n+1, n+1} v_{n}-U_{n, n+1} v_{n}\right\| \\
& \leq M \prod_{i=1}^{n} \sigma_{i},
\end{aligned}
$$

where $M$ is denote the possible different constants appearing in (3.22).

Hence

$$
\begin{aligned}
\left\|W_{n+1} v_{n+1}-W_{n} v_{n}\right\| & \leq\left\|W_{n+1} v_{n+1}-W_{n+1} v_{n}\right\|+\left\|W_{n+1} v_{n}-W_{n} v_{n}\right\| \\
& \leq\left\|v_{n+1}-v_{n}\right\|+M \prod_{i=1}^{n} \sigma_{i} .
\end{aligned}
$$

Substituting (3.23) into (3.21), we have

$$
\begin{aligned}
\left\|z_{n+1}-z_{n}\right\| \leq & \frac{\kappa \varrho_{n+1}}{1-\vartheta_{n+1}}\left\|u_{n+1}-u_{n}\right\| \\
& +\left|\frac{\varrho_{n+1}}{1-\vartheta_{n+1}}-\frac{\varrho_{n}}{1-\vartheta_{n}}\right|\left(\left\|f\left(u_{n}\right)\right\|+\left\|W_{n} v_{n}\right\|\right) \\
& +\frac{\theta_{n+1}}{1-\vartheta_{n+1}}\left\|v_{n+1}-v_{n}\right\|+\frac{M \theta_{n+1}}{1-\vartheta_{n+1}} \prod_{i=1}^{n} \sigma_{i} .
\end{aligned}
$$


Substituting (3.19) into (3.24), we get

$$
\begin{aligned}
\left\|z_{n+1}-z_{n}\right\| \leq & \frac{\kappa \varrho_{n+1}}{1-\vartheta_{n+1}}\left\|u_{n+1}-u_{n}\right\| \\
& +\left|\frac{\varrho_{n+1}}{1-\vartheta_{n+1}}-\frac{\varrho_{n}}{1-\vartheta_{n}}\right|\left(\left\|f\left(u_{n}\right)\right\|+\left\|W_{n} v_{n}\right\|\right) \\
& +\frac{\theta_{n+1}}{1-\vartheta_{n+1}}\left[\left\|u_{n+1}-u_{n}\right\|+\delta\|A\|\left(\frac{\left|\rho_{n+1}-\rho_{n}\right|}{\rho_{n+1}} \Omega_{n}\right)^{\frac{1}{2}}+\frac{\left|\mu_{n+1}-\mu_{n}\right|}{\mu_{n+1}} \Psi_{n+1}\right] \\
& +\frac{M \theta_{n+1}}{1-\vartheta_{n+1}} \prod_{i=1}^{n} \sigma_{i} \\
\leq & \frac{\kappa \varrho_{n+1}}{1-\vartheta_{n+1}}\left\|u_{n+1}-u_{n}\right\| \\
& +\left|\frac{\varrho_{n+1}}{1-\vartheta_{n+1}}-\frac{\varrho_{n}}{1-\vartheta_{n}}\right|\left(\left\|f\left(u_{n}\right)\right\|+\left\|W_{n} v_{n}\right\|\right) \\
& +\left\|u_{n+1}-u_{n}\right\|+\frac{\theta_{n+1}}{1-\vartheta_{n+1}} \delta\|A\|\left(\frac{\left|\rho_{n+1}-\rho_{n}\right|}{\rho_{n+1}} \Omega_{n}\right)^{\frac{1}{2}} \\
& +\frac{\theta_{n+1}}{1-\vartheta_{n+1}} \frac{\left|\mu_{n+1}-\mu_{n}\right|}{\mu_{n+1}} \Psi_{n+1}+\frac{M \theta_{n+1}}{1-\vartheta_{n+1}} \prod_{i=1}^{n} \sigma_{i} .
\end{aligned}
$$

From conditions $(C 1),(C 2)$ and $(C 3)$ imply that

$$
\limsup _{n \rightarrow \infty}\left(\left\|z_{n+1}-z_{n}\right\|-\left\|u_{n+1}-u_{n}\right\|\right) \leq 0 .
$$

Hence by Lemma 2.5, we obtain $\left\|z_{n}-u_{n}\right\| \rightarrow 0$ as $n \rightarrow \infty$. Consequently,

$$
\lim _{n \rightarrow \infty}\left\|u_{n+1}-u_{n}\right\|=\lim _{n \rightarrow \infty}\left(1-\vartheta_{n}\right)\left\|z_{n}-u_{n}\right\|=0 .
$$

From (3.19), (3.27), conditions $(C 1),(C 2)$ and $(C 3)$, we get

$$
\lim _{n \rightarrow \infty}\left\|v_{n+1}-v_{n}\right\|=0
$$

Since $u_{n+1}=\varrho_{n} f\left(u_{n}\right)+\vartheta_{n} u_{n}+\theta_{n} W_{n} v_{n}$, we have

$$
\begin{aligned}
\left\|u_{n}-W_{n} v_{n}\right\| & \leq\left\|u_{n}-u_{n+1}\right\|+\left\|u_{n+1}-W_{n} v_{n}\right\| \\
& \leq\left\|u_{n}-u_{n+1}\right\|+\varrho_{n}\left\|f\left(u_{n}\right)-W_{n} v_{n}\right\|+\vartheta_{n}\left\|u_{n}-W_{n} v_{n}\right\|,
\end{aligned}
$$

that is

$$
\left\|u_{n}-W_{n} v_{n}\right\| \leq \frac{1}{1-\vartheta_{n}}\left\|u_{n}-u_{n+1}\right\|+\frac{\varrho_{n}}{1-\vartheta_{n}}\left\|f\left(u_{n}\right)-W_{n} v_{n}\right\| .
$$

Since from (3.27), conditions $(C 1)$ and $(C 2)$, we get

$$
\lim _{n \rightarrow \infty}\left\|u_{n}-W_{n} v_{n}\right\|=0 .
$$

Now, using the definition of $u_{n+1}$, (3.16) and (3.17), we have

$$
\begin{aligned}
\left\|u_{n+1}-p\right\|^{2} \leq & \varrho_{n}\left\|f\left(u_{n}\right)-p\right\|^{2}+\vartheta_{n}\left\|u_{n}-p\right\|^{2}+\theta_{n}\left\|W_{n} v_{n}-p\right\|^{2} \\
\leq & \varrho_{n}\left\|f\left(u_{n}\right)-p\right\|^{2}+\vartheta_{n}\left\|u_{n}-p\right\|^{2}+\theta_{n}\left\|v_{n}-p\right\|^{2} \\
\leq & \varrho_{n}\left\|f\left(u_{n}\right)-p\right\|^{2}+\vartheta_{n}\left\|u_{n}-p\right\|^{2} \\
& +\theta_{n}\left[\left\|u_{n}-p\right\|^{2}+\delta\left(\delta-\frac{1}{L^{2}}\right)\left\|A^{*}\left(T_{\rho_{n}}^{\left(F_{2}, \psi_{2}\right)}-I\right) A u_{n}\right\|^{2}\right] \\
= & \varrho_{n}\left\|f\left(u_{n}\right)-p\right\|^{2}+\vartheta_{n}\left\|u_{n}-p\right\|^{2} \\
& +\left(1-\varrho_{n}-\vartheta_{n}\right)\left[\left\|u_{n}-p\right\|^{2}-\delta\left(\frac{1}{L^{2}}-\delta\right)\left\|A^{*}\left(T_{\rho_{n}}^{\left(F_{2}, \psi_{2}\right)}-I\right) A u_{n}\right\|^{2}\right]
\end{aligned}
$$


Therefore

$$
\begin{aligned}
& \left(1-\varrho_{n}-\vartheta_{n}\right) \delta\left(\frac{1}{L^{2}}-\delta\right)\left\|A^{*}\left(T_{\rho_{n}}^{\left(F_{2}, \psi_{2}\right)}-I\right) A u_{n}\right\|^{2} \\
\leq & \left\|u_{n}-p\right\|^{2}-\left\|u_{n+1}-u_{n}\right\|^{2}+\varrho_{n}\left\|f\left(u_{n}\right)-p\right\|^{2} \\
\leq & \left(\left\|u_{n}-p\right\|+\left\|u_{n+1}-p\right\|\right)\left\|u_{n}-u_{n+1}\right\|+\varrho_{n}\left\|f\left(u_{n}\right)-p\right\|^{2} .
\end{aligned}
$$

Since $\varrho_{n} \rightarrow 0$ and $\left\|u_{n+1}-u_{n}\right\| \rightarrow 0$ that

$$
\lim _{n \rightarrow \infty}\left\|A^{*}\left(T_{\rho_{n}}^{\left(F_{2}, \psi_{2}\right)}-I\right) A u_{n}\right\|^{2}=0 .
$$

Step 3. We claim that $\lim _{n \rightarrow \infty}\left\|u_{n}-v_{n}\right\|=0$ and $\lim _{n \rightarrow \infty}\left\|W v_{n}-v_{n}\right\|=0$.

Since $T_{\mu_{n}}^{\left(F_{1}, \psi_{1}\right)}$ is firmly nonexpansive and $I+\delta A^{*}\left(T_{\rho_{n}}^{\left(F_{2}, \psi_{2}\right)}-I\right) A$ is nonexpansive mapping, from the definition of $v_{n}$, we get that

$$
\begin{aligned}
& \left\|v_{n}-p\right\|^{2} \\
= & \left\|T_{\mu_{n}}^{\left(F_{1}, \psi_{1}\right)}\left(u_{n}+\delta A^{*}\left(T_{\rho_{n}}^{\left(F_{2}, \psi_{2}\right)}-I\right) A u_{n}\right)-T_{\mu_{n}}^{\left(F_{1}, \psi_{1}\right)} p\right\|^{2} \\
\leq & \left\langle v_{n}-p, u_{n}+\delta A^{*}\left(T_{\rho_{n}}^{\left(F_{2}, \psi_{2}\right)}-I\right) A u_{n}-p\right\rangle \\
= & \frac{1}{2}\left\{\left\|v_{n}-p\right\|^{2}+\left\|u_{n}+\delta A^{*}\left(T_{\rho_{n}}^{\left(F_{2}, \psi_{2}\right)}-I\right) A u_{n}-p\right\|^{2}\right. \\
& \left.-\left\|v_{n}-p-\left[u_{n}+\delta A^{*}\left(T_{\rho_{n}}^{\left(F_{2}, \psi_{2}\right)}-I\right) A u_{n}-p\right]\right\|^{2}\right\} \\
= & \frac{1}{2}\left\{\left\|v_{n}-p\right\|^{2}+\left\|\left(I+\delta A^{*}\left(T_{\rho_{n}}^{\left(F_{2}, \psi_{2}\right)}-I\right) A\right) u_{n}-\left(I+\delta A^{*}\left(T_{\rho_{n}}^{\left(F_{2}, \psi_{2}\right)}-I\right) A\right) p\right\|^{2}\right. \\
& \left.-\left\|v_{n}-u_{n}-\delta A^{*}\left(T_{\rho_{n}}^{\left(F_{2}, \psi_{2}\right)}-I\right) A u_{n}\right\|^{2}\right\} \\
\leq & \frac{1}{2}\left\{\left\|v_{n}-p\right\|^{2}+\left\|u_{n}-p\right\|^{2}-\left\|v_{n}-u_{n}-\delta A^{*}\left(T_{\rho_{n}}^{\left(F_{2}, \psi_{2}\right)}-I\right) A u_{n}\right\|^{2}\right\} \\
= & \frac{1}{2}\left\{\left\|v_{n}-p\right\|^{2}+\left\|u_{n}-p\right\|^{2}+\left[\left\|v_{n}-u_{n}\right\|^{2}+\delta^{2}\left\|A^{*}\left(T_{\rho_{n}}^{\left(F_{2}, \psi_{2}\right)}-I\right) A u_{n}\right\|^{2}\right.\right. \\
& \left.\left.-2 \delta\left\langle v_{n}-u_{n}, A^{*}\left(T_{\rho_{n}}^{\left(F_{2}, \psi_{2}\right)}-I\right) A u_{n}\right\rangle\right]\right\},
\end{aligned}
$$

which implies that

$$
\left\|v_{n}-p\right\|^{2} \leq\left\|u_{n}-p\right\|^{2}-\left\|v_{n}-u_{n}\right\|^{2}+2 \delta\left\|v_{n}-u_{n}\right\|\left\|A^{*}\left(T_{\rho_{n}}^{\left(F_{2}, \psi_{2}\right)}-I\right) A u_{n}\right\|,
$$

\section{From}

$$
\begin{aligned}
\left\|u_{n+1}-p\right\|^{2} \leq & \varrho_{n}\left\|f\left(u_{n}\right)-p\right\|^{2}+\vartheta_{n}\left\|u_{n}-p\right\|^{2}+\theta_{n}\left\|v_{n}-p\right\|^{2} \\
\leq & \varrho_{n}\left\|f\left(u_{n}\right)-p\right\|^{2}+\vartheta_{n}\left\|u_{n}-p\right\|^{2} \\
& +\theta_{n}\left[\left\|u_{n}-p\right\|^{2}-\left\|v_{n}-u_{n}\right\|^{2}+2 \delta\left\|v_{n}-u_{n}\right\|\left\|A^{*}\left(T_{\rho_{n}}^{\left(F_{2}, \psi_{2}\right)}-I\right) A u_{n}\right\|\right] \\
\leq & \varrho_{n}\left\|f\left(u_{n}\right)-p\right\|^{2}+\left(1-\varrho_{n}-\theta_{n}\right)\left\|u_{n}-p\right\|^{2} \\
& +\theta_{n}\left[\left\|u_{n}-p\right\|^{2}-\left\|v_{n}-u_{n}\right\|^{2}+2 \delta\left\|v_{n}-u_{n}\right\|\left\|A^{*}\left(T_{\rho_{n}}^{\left(F_{2}, \psi_{2}\right)}-I\right) A u_{n}\right\|\right] \\
\leq & \varrho_{n}\left\|f\left(u_{n}\right)-p\right\|^{2}+\left\|u_{n}-p\right\|^{2} \\
& -\theta_{n}\left\|v_{n}-u_{n}\right\|^{2}+2 \theta_{n} \delta\left\|v_{n}-u_{n}\right\|\left\|A^{*}\left(T_{\rho_{n}}^{\left(F_{2}, \psi_{2}\right)}-I\right) A u_{n}\right\|
\end{aligned}
$$


Therefore

$$
\begin{aligned}
\theta_{n}\left\|v_{n}-u_{n}\right\|^{2} \leq & \varrho_{n}\left\|f\left(u_{n}\right)-p\right\|^{2}+\left\|u_{n}-p\right\|^{2}-\left\|u_{n+1}-p\right\|^{2} \\
& +2 \theta_{n} \delta\left\|v_{n}-u_{n}\right\|\left\|A^{*}\left(T_{\rho_{n}}^{\left(F_{2}, \psi_{2}\right)}-I\right) A u_{n}\right\| \\
\leq & \varrho_{n}\left\|f\left(u_{n}\right)-p\right\|^{2}+\left\|u_{n}-u_{n+1}\right\|\left(\left\|u_{n}-p\right\|+\left\|u_{n+1}-p\right\|\right) \\
& +2 \theta_{n} \delta\left\|v_{n}-u_{n}\right\|\left\|A^{*}\left(T_{\rho_{n}}^{\left(F_{2}, \psi_{2}\right)}-I\right) A u_{n}\right\|
\end{aligned}
$$

Since $\varrho_{n} \rightarrow 0,\left\|u_{n}-u_{n+1}\right\| \rightarrow 0,\left\|A^{*}\left(T_{\rho_{n}}^{\left(F_{2}, \psi_{2}\right)}-I\right) A u_{n}\right\| \rightarrow 0$ and it is easily seen that $\liminf _{n \rightarrow \infty} \theta_{n}>0$, so we have

$$
\lim _{n \rightarrow \infty}\left\|v_{n}-u_{n}\right\|=0
$$

From $\left\|W_{n} v_{n}-v_{n}\right\| \leq\left\|W_{n} v_{n}-u_{n}\right\|+\left\|u_{n}-v_{n}\right\|$, we also have $\left\|W_{n} v_{n}-v_{n}\right\| \rightarrow 0$. At the same time, we note that

$$
\left\|W v_{n}-v_{n}\right\| \leq\left\|W v_{n}-W_{n} v_{n}\right\|+\left\|W_{n} v_{n}-v_{n}\right\| .
$$

It follows from (3.39) and Remark 2.1 that

$$
\lim _{n \rightarrow \infty}\left\|W v_{n}-v_{n}\right\|=0 \text {. }
$$

Step 4. We show that $\left\{u_{n}\right\}$ generated by Algorithm 1 converges strongly to $u^{*} \in$ $\cap_{i=1}^{\infty} \operatorname{Fix}\left(K_{i}\right) \cap \Gamma$, where $u^{*}=P_{\cap_{i=1}^{\infty} F i x\left(K_{i}\right) \cap \Gamma} f\left(u^{*}\right)$. To that end, we show that

$$
\limsup _{n \rightarrow \infty}\left\langle f\left(u^{*}\right)-u^{*}, u_{n}-u^{*}\right\rangle \leq 0 .
$$

where $u^{*}=P_{\cap_{i=1}^{\infty} F i x\left(K_{i}\right) \cap \Gamma} f\left(u^{*}\right)$. First, we choose a subsequence $\left\{v_{n_{j}}\right\}$ of $\left\{v_{n}\right\}$ such that

$$
\lim _{j \rightarrow \infty}\left\langle f(z)-z, v_{n_{j}}-z\right\rangle=\limsup _{n \rightarrow \infty}\left\langle f(z)-z, v_{n}-z\right\rangle .
$$

Step 4.1 We show that $u^{*} \in \cap_{i=1}^{\infty} \operatorname{Fix}\left(K_{i}\right) \cap \Gamma$.

Since $\left\{v_{n_{j}}\right\}$ is bounded, there exists a subsequence $\left\{v_{n_{j_{i}}}\right\}$ of $\left\{v_{n_{j}}\right\}$, which converges weakly to $u^{*}$. Without loss of generality, we can assume that $v_{n_{j}} \rightarrow u^{*}$. From $\| W v_{n}-$ $v_{n} \| \rightarrow 0$, we obtain $W v_{n_{j}} \rightarrow u^{*}$.

Next, we will show $u^{*} \in F i x(W)$. Assume that $u^{*} \neq F i x(W)$. Since $v_{n_{j}} \rightarrow u^{*}$ and $u^{*} \neq W u^{*}$, from Opail's condition, we have

$$
\begin{aligned}
\liminf _{j \rightarrow \infty}\left\|v_{n_{j}}-u^{*}\right\| & <\liminf _{j \rightarrow \infty}\left\|v_{n_{j}}-W u^{*}\right\| \\
& \leq \liminf _{j \rightarrow \infty}\left(\left\|v_{n_{j}}-W v_{n_{j}}\right\|+\left\|W v_{n_{j}}-W u^{*}\right\|\right) \\
& \leq \liminf _{j \rightarrow \infty}\left\|v_{n_{j}}-u^{*}\right\| .
\end{aligned}
$$

This is a contradiction. So we get $u^{*} \in F i x(W)=\cap_{i=1}^{\infty} F i x\left(K_{i}\right)$.

Now, we show $u^{*} \in \Gamma$. Since $v_{n}=T_{\mu_{n}}^{\left(F_{1}, \psi_{1}\right)}\left(u_{n}+\delta A^{*}\left(T_{\rho_{n}}^{\left(F_{2}, \psi_{2}\right)}-I\right) A u_{n}\right)$, we have

$$
F_{1}\left(v_{n}, v\right)+\psi_{1}\left(v_{n}, v\right)+\frac{1}{\mu_{n}}\left\langle v-v_{n}, v_{n}-d_{n}\right\rangle \geq 0, \forall v \in C,
$$

where $d_{n}=u_{n}+\delta A^{*}\left(T_{\rho_{n}}^{\left(F_{2}, \psi_{2}\right)}-I\right) A u_{n}$. It follows from the monotonicity property of $F_{1}$ that

$$
\psi_{1}\left(v_{n}, v\right)+\frac{1}{\mu_{n}}\left\langle v-v_{n}, v_{n}-d_{n}\right\rangle \geq F_{1}\left(v, v_{n}\right), \forall v \in C
$$

which implies that

$$
\psi_{1}\left(v_{n}, v\right)+\left\langle v-v_{n_{i}}, \frac{v_{n_{i}}-u_{n_{i}}}{\mu_{n}}+\delta A^{*}\left(\frac{\left(T_{a_{n_{i}}}^{\left(F_{2}, \psi_{2}\right)}-I\right) A u_{n_{i}}}{\mu_{n}}\right)\right\rangle \geq F_{1}\left(v, v_{n_{i}}\right), \forall v \in C
$$


From $\left\|v_{n}-u_{n}\right\| \rightarrow 0$, we get $v_{n_{i}} \rightarrow u^{*}$ and $\frac{v_{n_{i}}-u_{n_{i}}}{\mu_{n}} \rightarrow 0$. Since $\lim _{n \rightarrow \infty} \| A^{*}\left(T_{\rho_{n}}^{\left(F_{2}, \psi_{2}\right)}-\right.$ I) $A u_{n} \|=0$, then $A^{*}\left(\frac{\left.T_{a_{n_{i}}}^{\left(F_{2}, \psi_{2}\right)}-I\right) A u_{n_{i}}}{\mu_{n}}\right) \rightarrow 0$. Therefore

$$
\psi_{1}\left(v_{n_{i}}, v\right) \geq F_{1}\left(v, v_{n_{i}}\right), \psi_{1}\left(u^{*}, v\right) \geq F_{1}\left(v, u^{*}\right) .
$$

Let $v_{t}=t v+(1-t) u^{*}$ for all $t \in(0,1]$. Since $v \in C$ and $u^{*} \in C$, we get $v_{t} \in C$. It follows from Assumption 2.1 that

$$
\begin{aligned}
0=F_{1}\left(v_{t}, v_{t}\right)+\psi_{1}\left(v_{t}, v_{t}\right) \leq & t F_{1}\left(v_{t}, v\right)+(1-t) F_{1}\left(v_{t}, u^{*}\right) \\
& +t \psi_{1}\left(v_{t}, v\right)+(1-t) \psi_{1}\left(v_{t}, u^{*}\right) \\
= & t\left(F_{1}\left(v_{t}, v\right)+\psi_{1}\left(v_{t}, u^{*}\right)\right) \\
& +(1-t)\left(F_{1}\left(v_{t}, u^{*}\right)+\psi_{1}\left(v_{t}, u^{*}\right)\right) \\
\leq & F_{1}\left(v_{t}, v\right)+\psi_{1}\left(v_{t}, v\right),
\end{aligned}
$$

so $0 \leq F_{1}\left(v_{t}, v\right)+\psi_{1}\left(v_{t}, v\right)$. Letting $t \rightarrow 0$, se obtain $0 \leq F_{1}\left(u^{*}, v\right)+\psi_{1}\left(u^{*}, v\right)$. This implies that $u^{*} \in \operatorname{GEP}\left(F_{1}, \psi_{1}\right)$.

Next, we show that $A u^{*} \in \operatorname{GEP}\left(F_{2}, \psi_{2}\right)$. Since $\left\|v_{n}-u_{n}\right\| \rightarrow 0, v_{n} \rightarrow u^{*}$ as $n \rightarrow \infty$ and $\left\{u_{n}\right\}$ is bounded, there exists a subsequence $\left\{u_{n_{j}}\right\}$ of $\left\{u_{n}\right\}$ such that $u_{n_{j}} \rightarrow u^{*}$ and since $A$ is bounded linear operator, thus, $A u_{n_{j}} \rightarrow A u^{*}$. From $\left.\| T_{\rho_{n}}^{\left(F_{2}, \psi_{2}\right)}-I\right) A u_{n} \| \rightarrow 0$, we have $T_{\rho_{n}}^{\left(F_{2}, \psi_{2}\right)} A u_{n_{j}} \rightarrow A u^{*}$. Therefore from Lemma 2.2, we have

$F_{2}\left(T_{\rho_{n_{j}}}^{\left(F_{2}, \psi_{2}\right)} A u_{n_{j}}, v\right)+\psi_{2}\left(T_{\rho_{n_{j}}}^{\left(F_{2}, \psi_{2}\right)} A u_{n_{j}}, v\right)+\frac{1}{\rho_{n_{j}}}\left\langle v-T_{\rho_{n_{j}}}^{\left(F_{2}, \psi_{2}\right)} A u_{n_{j}}, T_{\rho_{n_{j}}}^{\left(F_{2}, \psi_{2}\right)} A u_{n_{j}}-A u^{*}\right\rangle \geq 0$,

$\forall v \in Q$. Since $F_{2}$ is upper semicontinuous in first argument, from above inequality, we obtain

$$
F_{2}\left(A u^{*}, v\right)+\psi_{2}\left(A u^{*}, v\right) \geq 0, \forall v \in Q,
$$

which mean that $A u^{*} \in G E P\left(F_{2}, \psi_{2}\right)$ and hence $u^{*} \in \Gamma$. So, we get $u^{*} \in \cap_{i=1}^{\infty} F i x\left(K_{i}\right) \cap \Gamma$.

Step 4.2 We show that $\left\{u_{n}\right\}$ convergence strongly to $u^{*} \in \cap_{i=1}^{\infty} \operatorname{Fix}\left(K_{i}\right) \cap \Gamma$, where $u^{*}=P_{\cap_{i=1}^{\infty} F i x\left(K_{i}\right) \cap \Gamma} f\left(u^{*}\right)$.

Since $u^{*}=P_{\cap_{i=1}^{\infty}} \operatorname{Fix}\left(K_{i}\right) \cap \Gamma f\left(u^{*}\right)$, we have

$$
\begin{aligned}
\limsup _{n \rightarrow \infty}\left\langle f\left(u^{*}\right)-u^{*}, u_{n}-u^{*}\right\rangle & =\lim _{j \rightarrow \infty}\left\langle f\left(u^{*}\right)-u^{*}, u_{n_{j}}-u^{*}\right\rangle \\
& =\lim _{j \rightarrow \infty}\left\langle f\left(u^{*}\right)-u^{*}, v_{n_{j}}-u^{*}\right\rangle \\
& =\left\langle f\left(u^{*}\right)-u^{*}, u^{*}-u^{*}\right\rangle \leq 0
\end{aligned}
$$

From the definition of $u_{n+1}$ in Algorithm 1, we get that

$$
\begin{aligned}
& \left\|u_{n+1}-u^{*}\right\|^{2} \\
\leq & \left\|\vartheta_{n}\left(u_{n}-u^{*}\right)+\theta_{n}\left(W_{n} v_{n}-u^{*}\right)\right\|^{2}+2 \varrho_{n}\left\langle f\left(u_{n}\right)-u^{*}, u_{n+1}-u^{*}\right\rangle \\
= & \left\{\vartheta_{n}\left\|u_{n}-u^{*}\right\|+\theta_{n}\left\|W_{n} v_{n}-u^{*}\right\|\right\}^{2}+2 \varrho_{n}\left\langle f\left(u_{n}\right)-f\left(u^{*}\right)+f\left(u^{*}\right)-u^{*}, u_{n+1}-u^{*}\right\rangle \\
\leq & \left\{\vartheta_{n}\left\|u_{n}-u^{*}\right\|+\theta_{n}\left\|W_{n} v_{n}-u^{*}\right\|\right\}^{2}+2 \varrho_{n}\left\langle f\left(u^{*}\right)-u^{*}, u_{n+1}-u^{*}\right\rangle \\
& +2 \varrho_{n}\left\langle f\left(u_{n}\right)-f\left(u^{*}\right), u_{n+1}-u^{*}\right\rangle \\
\leq & \left\{\vartheta_{n}\left\|u_{n}-u^{*}\right\|+\theta_{n}\left\|v_{n}-u^{*}\right\|\right\}^{2}+2 \kappa \varrho_{n}\left\|u_{n}-u^{*}\right\|\left\|u_{n+1}-u^{*}\right\| \\
& +2 \varrho_{n}\left\langle f\left(u_{n}\right)-u^{*}, u_{n+1}-u^{*}\right\rangle \\
\leq & \left(1-\varrho_{n}\right)^{2}\left\|u_{n}-u^{*}\right\|^{2}+\kappa \varrho_{n}\left(\left\|u_{n+1}-u^{*}\right\|^{2}+\left\|u_{n}-u^{*}\right\|^{2}\right) \\
(3.52) & +2 \varrho_{n}\left\langle f\left(u^{*}\right)-u^{*}, u_{n+1}-u^{*}\right\rangle,
\end{aligned}
$$


which implies that

$$
\begin{aligned}
\left\|u_{n+1}-u^{*}\right\|^{2} \leq & \frac{\left(1-\varrho_{n}\right)^{2}+\kappa \varrho_{n}}{1-\kappa \varrho_{n}}\left\|u_{n}-u^{*}\right\|^{2}+\frac{2 \varrho_{n}}{1-\kappa \varrho_{n}}\left\langle f\left(u^{*}\right)-u^{*}, u_{n+1}-u^{*}\right\rangle \\
= & \frac{1-2 \varrho_{n}+\kappa \varrho_{n}}{1-\kappa \varrho_{n}}\left\|u_{n}-u^{*}\right\|^{2}+\frac{\varrho_{n}^{2}}{1-\kappa \varrho_{n}}\left\|u_{n}-u^{*}\right\|^{2} \\
& +\frac{2 \varrho_{n}}{1-\kappa \varrho_{n}}\left\langle f\left(u^{*}\right)-u^{*}, u_{n+1}-u^{*}\right\rangle \\
\leq & \left\{1-\frac{2(1-\kappa) \varrho_{n}}{1-\kappa \varrho_{n}}\right\}\left\|u_{n}-u^{*}\right\|^{2}+\frac{2(1-\kappa) \varrho_{n}}{1-\kappa \varrho_{n}} \\
& \times\left\{\frac{M \varrho_{n}}{2(1-\kappa)}+\frac{1}{1-\kappa}\left\langle f\left(u^{*}\right)-u^{*}, u_{n+1}-u^{*}\right\rangle\right\} \\
= & \left(1-\varphi_{n}\right)\left\|u_{n}-u^{*}\right\|^{2}+\phi_{n} \varphi_{n},
\end{aligned}
$$

where $\varphi_{n}=\frac{2(1-\kappa) \varrho_{n}}{1-\kappa \varrho_{n}}$ and $\phi_{n}=\frac{M \varrho_{n}}{2(1-\kappa)}+\frac{1}{1-\kappa}\left\langle f\left(u^{*}\right)-u^{*}, u_{n+1}-u^{*}\right\rangle$. It easily seen that $\sum_{n=0}^{\infty} \varphi_{n}=\infty$ and $\lim \sup _{n \rightarrow \infty} \phi_{n} \leq 0$. Now applying Lemma 2.6, (3.51), (3.52) and (3.53), we conclude that $u_{n} \rightarrow u^{*}$ as $n \rightarrow \infty$. Consequently, from (3.38), we have $v_{n} \rightarrow u^{*}$ as $n \rightarrow \infty$. This completes the proof.

For each $i=1,2, \cdots$, setting the infinite family $K_{i}$ of nonexpansive mappings to be identity mappings. We obtain the following algorithm for solving split generalized equilibrium problem.

Initialization: Choose the sequence $\left\{\varrho_{n}\right\},\left\{\theta_{n}\right\},\left\{\vartheta_{n}\right\}$ in $(0,1)$ such that C1-C2 are satisfied and $\delta \in\left(0, \frac{1}{L^{2}}\right), L$ is the spectral radius of the operator $A^{*} A$. Given $\left\{\mu_{n}\right\} \subset[\mu, \infty)$ with $\mu>0,\left\{\rho_{n}\right\} \subset[\rho, \infty)$ with $\rho>0$ such that C3 is satisfied.

Iterative Steps: For $u_{n} \in H_{1}$, Set $v_{n}$ as:

$$
v_{n}=T_{\rho_{n}}^{\left(F_{1}, \psi_{1}\right)}\left(u_{n}+\delta A^{*}\left(T_{\rho_{n}}^{\left(F_{2}, \psi_{2}\right)}-I\right) A u_{n}\right),
$$

Step 2. Compute

$$
u_{n+1}=\varrho_{n} f\left(u_{n}\right)+\vartheta_{n} u_{n}+\theta_{n} v_{n},
$$

If $v_{n}=u_{n}$, stop $v_{n}$ is the solution of the SGEP Problem, otherwise set $n:=n+1$ and go back to Step 1 .

Corollary 3.1. Let $C \subset H_{1}, Q \subset H_{2}$ be nonempty closed and convex subsets of real Hilbert space $H_{1}$ and $H_{2}$ respectively. Suppose $F_{1}, \psi_{1}: C \times C \rightarrow \mathbb{R}$ and $F_{2}, \psi_{2}: Q \times Q \rightarrow \mathbb{R}$ be nonlinear mappings satisfying Assumption 2.1 with $F_{2}$ as upper semicontinuous in the first argument. Assume that the solution set $\Gamma \neq \emptyset$. Also, let $f: H_{1} \rightarrow H_{1}$ be a contraction mapping with constant $\kappa \in(0,1), A: H_{1} \rightarrow H_{2}$ be a bounded linear operator with adjoint $A^{*}$. Suppose that $\left\{\varrho_{n}\right\},\left\{\vartheta_{n}\right\}$ and $\left\{\theta_{n}\right\}$ are three sequences in $(0,1)$ such that $\varrho_{n}+\vartheta_{n}+\theta_{n}=1$. For given $u_{0} \in C$ arbitrary, the sequence $\left\{u_{n}\right\}$ generated by the above algorithm converges strongly to $u^{*}=P_{\Gamma} f\left(u^{*}\right)$

Proof. Take $K_{i} u=u$ for all $i=1,2, \ldots$ and for all $u \in C W_{n} u=u$ in Theorem 3.2. Hence, the proof follows immediately from Theorem 3.2.

\section{NUMERICAL ILLUSTRATIONS}

In this section, we give some numerical examples to illustrate the computational performance of the proposed algorithm. 
Example 4.1. Suppose that $H_{1}=H_{2}=\mathbb{R}^{3}$ and $C=Q=[0,10] \times[0,10] \times[0,10]$. Suppose for each $u \in H_{1}, A: H_{1} \rightarrow H_{2}$ be defined by $A u=\frac{u}{5}$ and for each $u \in C, i=1,2, \cdots$, we define $K_{i}: C \rightarrow H$ as

$$
K_{i}(u)=\frac{u+2 i}{1+3 i} .
$$

It can be easily seen that $K_{i}$ is a nonexpansive mapping for each $i \in \mathbb{N}$. and $F\left(K_{i}\right)=\left\{\frac{2}{3}\right\}$. For each $u, v \in C$, define the bifunctions $F_{1}, \psi_{1}: C \times C \rightarrow \mathbb{R}$ by $F_{1}(u, v)=2(u+1)(v-u)$ and $\psi_{1}(u, v)=u(v-u)$. Also for each $\bar{u}, \bar{v} \in Q$, define $F_{2}, \psi_{2}: Q \times Q \rightarrow \mathbb{R}$ by $F_{2}(\bar{u}, \bar{v})=$ $(\bar{u}-10)(\bar{v}-\bar{u})$ and $\psi_{2}(\bar{u}, \bar{v})=\bar{u}-\bar{v}$. It is easy to check that

$$
T_{\mu_{n}}^{F_{1}, \psi_{1}} u=\frac{u-2 \mu_{n}}{3 \mu_{n}+1}
$$

and

$$
T_{\mu_{n}}^{F_{2}, \psi_{2}} \bar{u}=\frac{\bar{u}+11 \mu_{n}}{\mu_{n}+1} .
$$

We define the contraction map $f(u)=\frac{u}{2}$ with constant $\kappa=0.5 . \mu_{n}=\frac{n}{n+1}, \varrho_{n}=$ $\frac{1}{n+1}, \vartheta_{n}=\frac{n}{2 n+3}, \theta_{n}=1-\varrho_{n}-\vartheta_{n}, \eta_{n}=\frac{1}{n+1}, \zeta_{n}=\frac{1}{(n+1)^{2}}$ and $\delta=\frac{1}{\|A\|^{2}}$ as the control parameters for the numerical illustrations.

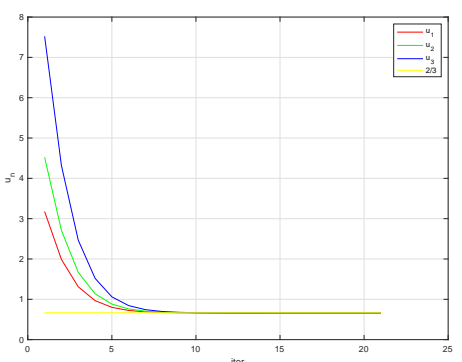

(A) Case I: Convergence of $u_{n}$

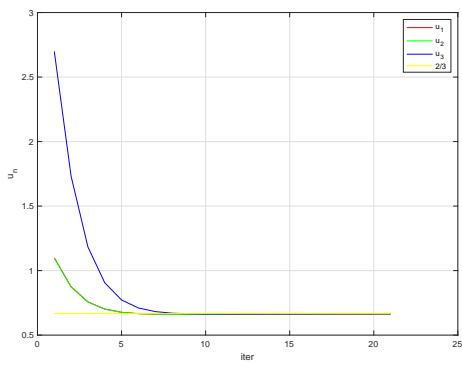

(C) Case II: Convergence of $u_{n}$

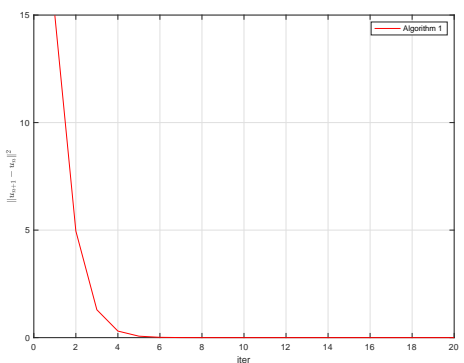

(B) Case I: Error against number of Iterations

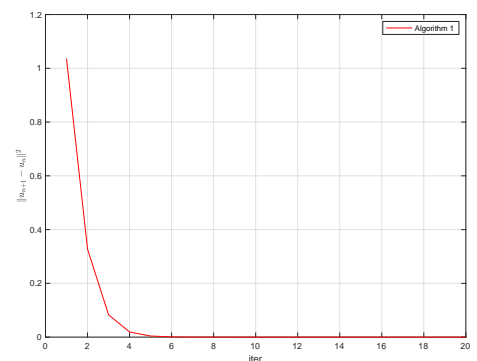

(D) Case II: Error against number of Iterations

FIGURE 1. Convergence of the sequence $u_{n}$ with different initial points.

In Figure 1, we illustrate the convergence of the sequence generated by Algorithm 1 by considering four different cases of initial points randomly generated in $[0,10]$. It can be seen that the iterates of the proposed algorithm converges to the common solution of the considered problem (1.4)-(1.5). 
TABLE 1. Comparative results of Algorithm 1 and the Scheme 1.9 (Algorithm 2) with different initial values and tolerance

\begin{tabular}{lrrrrr}
\hline TOL & \multicolumn{1}{l}{$x_{0}$} & \multicolumn{2}{c}{ Algorithm 1 } & \multicolumn{2}{c}{ Algorithm 2 } \\
& & Iter. & CPU & Iter. & CPU \\
\hline $10^{-6}$ & $(-3,4,-1)$ & 31 & 0.0504 & 46 & 0.0646 \\
& $(4,0,2)$ & 31 & 0.0678 & 48 & 0.1058 \\
& $(5,-8,1)$ & 31 & 0.0414 & 48 & 0.1104 \\
\hline $10^{-7}$ & $(-3,4,-1)$ & 57 & 0.0418 & 81 & 0.1103 \\
& $(4,0,2)$ & 57 & 0.0341 & 83 & 0.1042 \\
& $(5,-8,1)$ & 57 & 0.0372 & 81 & 0.0867 \\
\hline $10^{-8}$ & $(-3,4,-1)$ & 109 & 0.0357 & 142 & 0.0997 \\
& $(4,0,2)$ & 109 & 0.0311 & 144 & 0.1145 \\
& $(5,-8,1)$ & 109 & 0.0365 & 143 & 0.0948 \\
\hline
\end{tabular}

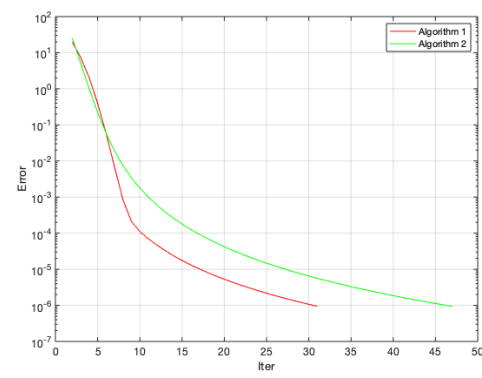

(A) Case I: $u_{0}=(-3,4,-1)$

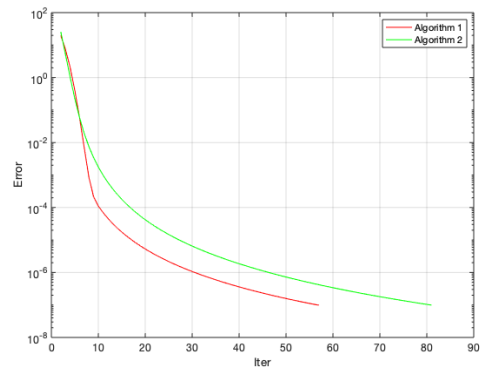

(B) Case II: $u_{0}=(4,0,2)$

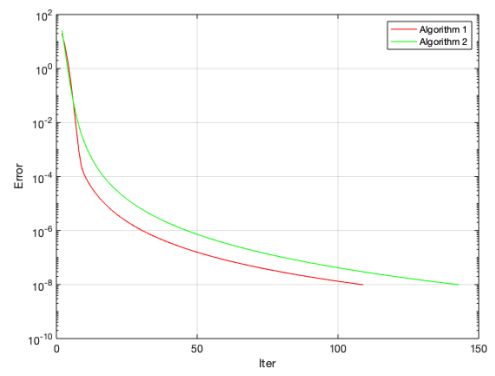

(C) Case III: $u_{0}=(5,-8,1)$

FIGURE 2. Example 4.1, compared results for different initial values with tolerance $10^{-8}$.

It can be observed from Table 1 that, for the given tolerances, the choices of inertial points do not affect the convergence of the proposed Algorithm. The proposed algorithm competes well and performs better than the compared algorithm in terms of less number of iterations and computational time.

Next, we present an example in infinite dimensional space to support our strong convergence result. We reported the computational performance of the proposed algorithm in Figure 3 and the comparative results in Figure 4. 
Example 4.2. Suppose $H_{1}=H_{2}=l_{2}(\mathbb{R})=\left\{u=\left(u_{1}, u_{2}, \cdots, u_{t}, \cdots\right), u_{t} \in \mathbb{R}\right.$ and $\left.\sum_{t=1}^{\infty}\left|u_{t}\right|^{2}<\infty\right\}$ be the vector space whose elements are 2-summable sequences $\left\{u_{t}\right\}_{t=1}^{\infty}$ of scalars in $\mathbb{R}$. with inner product $\langle\cdot, \cdot\rangle: l_{2} \times l_{2} \rightarrow \mathbb{R}$ and induced norm $\|\cdot\|: l_{2} \rightarrow$ $\mathbb{R}$ defined for each $u=\left\{u_{t}\right\}_{t=1}^{\infty}, v=\left\{v_{t}\right\}_{t=1}^{\infty} \in l_{2}$ as $\langle u, v\rangle=\sum_{k=1}^{\infty} u_{t} v_{t}$ and $\|u\|=$ $\sqrt{\sum_{t=1}^{\infty}\left|u_{t}\right|^{2}}$ respectively. Suppose the bounded linear operator $A: l_{2} \rightarrow l_{2}$ be defined by $A u=\left(\frac{u_{1}}{3}, \frac{u_{2}}{3}, \cdots, \frac{u_{t}}{3}, \cdots\right)$ for any $\left\{u_{t}\right\}_{t=1}^{\infty} \in l_{2}$, therefore, the adjoint is $A^{*} v=$ $\left(\frac{v_{1}}{3}, \frac{v_{2}}{3}, \cdots, \frac{v_{t}}{3}, \cdots\right)$ for each $\left\{v_{t}\right\}_{t=1}^{\infty} \in l_{2}$. We set the sets $C=\left\{u \in l_{2}:\|u\| \leq 1\right\}$ and $Q=$ $\left\{v \in l_{2}:\|v\| \leq 1\right\}$. For each $\left\{u_{t}\right\}_{t=1}^{\infty},\left\{v_{t}\right\}_{t=1}^{\infty} \in C$, we define the bifunctions $F_{1}, \psi_{1}: C \times$ $C \rightarrow \mathbb{R}$ by $F_{1}(u, v)=v^{2}+3 u v-4 v^{2}$ and $\psi_{1}(u, v)=v^{2}-u^{2}$. Also for each $\left\{\bar{u}_{t}\right\}_{t=1}^{\infty},\left\{\bar{v}_{t}\right\}_{t=1}^{\infty} \in$ $Q$, define $F_{2}, \psi_{2}: Q \times Q \rightarrow \mathbb{R}$ by $F_{2}(\bar{u}, \bar{v})=2 \bar{v}^{2}+\bar{u} \bar{v}+3 \bar{u}^{2}$ and $\psi_{1}(u, \bar{v})=\bar{u}-\bar{v}$. It is easy to check that

$$
T_{\mu_{n}}^{F_{1}, \psi_{1}} u=\frac{u}{7 \mu_{n}+1} \quad \text { and } \quad T_{\mu_{n}}^{F_{2}, \psi_{2}} \bar{u}=\frac{\bar{u}+\mu_{n}}{5 \mu_{n}+1} .
$$

For each $i=1,2, \cdots$, we define $K_{i}: C \rightarrow H$ as

$$
K_{i}=\frac{u+2 i}{1+3 i}
$$

for all $\left\{u_{t}\right\}_{t=1}^{\infty} \in C$.

For this example, for all $\left\{u_{t}\right\}_{t=1}^{\infty} \in C$, we define the contraction map $f(u)=\frac{u}{2}$. We set the control parameters the same as in Example 4.1. We present the computational result for two different initial points $u_{0}=(1,2, \ldots, 1000,0, \ldots)^{T}$ and $\left(1,1, \ldots, 1_{1000}, 0, \ldots\right)^{T}$.

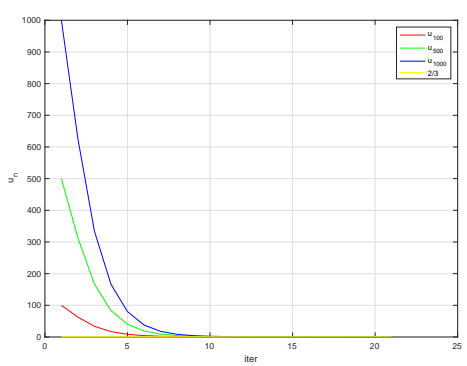

(A) Case I: Convergence of $u_{n}$

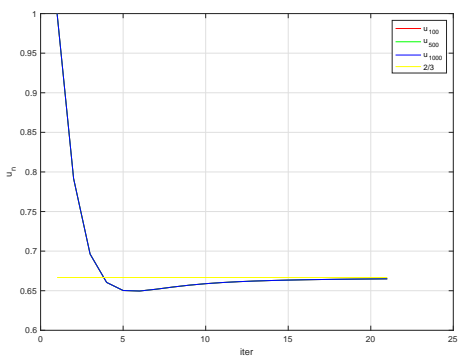

(C) Case II: Convergence of $u_{n}$

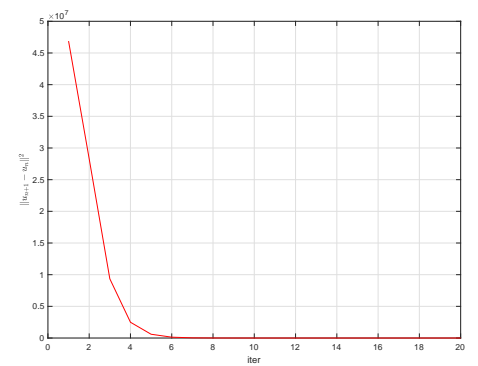

(B) Case I: Error against number of Iterations

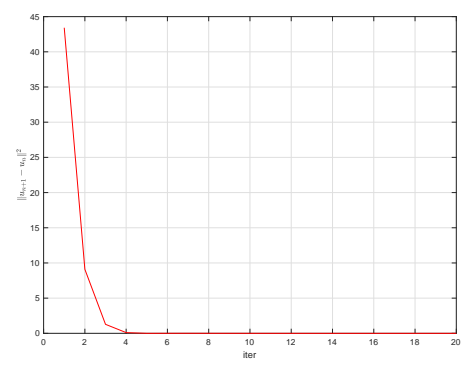

(D) Case II: Error against number of Iterations

FIGURE 3. Convergence of the sequence $u_{n}$ with different initial points. 


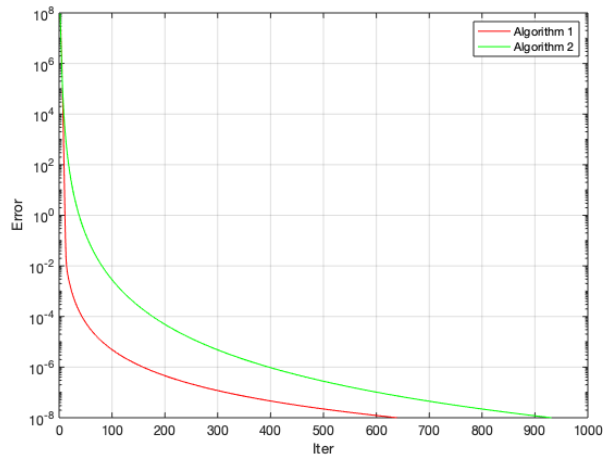

(A) Case I: $u_{0}=(1,2, \ldots, 1000,0, \ldots)^{T}$

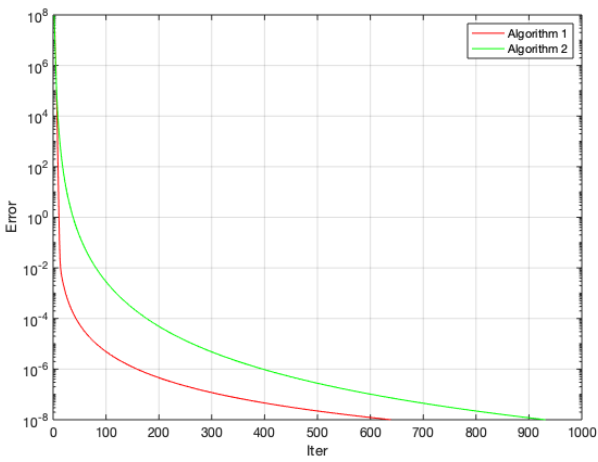

(B) Case II: $\left(1,1, \ldots, 1_{1000}, 0, \ldots\right)^{T}$.

FIGURE 4. Example 4.2, compared results for two different initial values with tolerance $10^{-8}$.

\section{CONCLUSION}

In this paper, an iterative method for solving a common solution of a split generalized equilibrium problem and a fixed point of a set of family of an infinite nonexpansive mappings in real Hilbert space is proposed and studied. Under some suitable and easy to verify conditions, a strong convergence theorem of the sequences generated by the proposed method to the common element of the solutions of the considered problems based on the viscosity method are established. Numerical examples to illustrate the implementation of the proposed method indicates that, the proposed method is implementable and our theorem is an extension and improvement of some results obtained in the literature. As a future work, we are going to consider a self-adaptive version of the proposed method that does not require the computation of the operator norms involved.

Acknowledgments. The authors thank the referees for comments and suggestions on this manuscript. The corresponding author would like to thank King Mongkut's University of Technology North Bangkok (KMUTNB), Rayong Campus. This research was funded by King Mongkut's University of Technology North Bangkok. Contract no. KMUTNB63-KNOW-016.

\section{REFERENCES}

[1] Boț, R. I.; Csetnek, E. R.; Hendrich, C. Inertial Douglas-Rachford splitting for monotone inclusion problems. Appl. Math. Comput. 256 (2015), 472-487.

[2] Censor, Y.; Elfving, T. A multiprojection algorithm using Bregman projections in product space. Numer. Algorithm. 8 (1994), 221-239.

[3] Cianciaruso, F.; Marino, G.; Muglia, L.; Yao, Y. A hybrid projection algorithm for finding solution of mixed equilibrium problem and variational inequality problem. Fixed Point Theory Appl. 2010 (2010), Article ID 383740, 19 pages, doi:10.1155/2010/383740.

[4] Combettes, P. L.; Hirstoaga, S. A. Equilibrium programming in Hilbert spaces. J Nonlinear Convex Anal. 6 (2005), no. 1, 117-136.

[5] Hey, I.; Bnouhachem, A.; Rassias, T. M. An Iterative Method for a Common Solution of Split Generalized Equilibrium Problems and Fixed Points of a Finite Family of Nonexpansive Mapping. Nonlinear Analysis and Global Optimization. 21 (2021), no. 10, 2237-2255.

[6] Kazmi, K. R.; Rizvi, S. H. Iterative approximation of a common solution of a split equilibrium problem, a variational inequality problem and a fixed point problem. J. Egypt. Math. Soc. 21 (2013), no. 1, 44-51. 
[7] Kazmi, K. R.; Rizvi, S. H. Iterative approximation of a common solution of a split generalized equilibrium problem and a fixed point problem for nonexpansive semigroup. Mathematical Sciences. 7 (2013), no. 1.

[8] Kazmi, K. R.; Rizvi, S. H. Iterative approximation of a common solution of a split equilibrium problem, a variational inequality problem and a fixed point problem. J. Egypt. Math. Soc. 21 (2013), no. 1, 44-51.

[9] Krasnosel'skiı M. A. Two remarks on the method of successive approximations. Usp. Mat. Nauk 10 (1955), no. 1, 123-127.

[10] Maingé, P.-E. Convergence theorems for inertial KM-type algorithms. J. Comput. Appl. Math. 219 (2010), no. 1, 223-236.

[11] Moudafi, A. Viscosity approximation methods for fixed-points problems. J. Math. Anal. Appl. 241 (2000), 46-55.

[12] Opial, Z. Weak convergence of the sequence of successive approximations for nonexpansive mappings. Bull. Am. Math. Soc. 73 (1967), 591-597.

[13] Sahebi, H. R.; Razani, A. An iterative algorithm for finding the solution of a general equilibrium problem system. Filomat 28 (2014), no. 7, 1393-1415.

[14] Sahebi, H. R.; Ebrahimi, S. A Viscosity iterative algorithm for the optimization problem system. Filomat 31 (2017), no. 8, 2249-2266.

[15] Shimoji, K.; Takahashi, W. Strong convergence to common fixed points of infinite nonexpansive mappings and applications. Taiwan. J. Math. 5 (2001), no. 2, 387-404.

[16] Suntai, S.; Cholamjiak, P.; Cho, Y.-J.; Cholamjiak, W. On solving split equilibrium problems and fixed point problems of nonspreading multi-valued mappings in Hilbert space. Fixed Point Theory Appl. (2016), Article number: 35 .

[17] Suzuki, T. Strong convergence of Krasnoselskii and Mann's type sequences for one-parameter nonexpansive semigroups without Bochner integrals. J. Math. Anal. Appl. 305 (2005), no. 1, 227-239.

[18] Takahashi, S.; Takahashi, W. Viscosity approximation methods for equilibrium problems and fixed point problems in Hilbert spaces. J. Math. Anal. Appl. 331 (2007), no. 1, 506-515.

[19] Takahashi, W. Nonlinear Functional Analysis, Yokohama Publishers, Yokohama, Japan, 2000.

[20] Takahashi, S.; Takahashi, W. Strong convergence theorem for a generalized equilibrium problem and a nonexpansive mapping in a Hilbert space. Nonlinear Anal. 69 (2008), 1025-1033.

[21] Takahashi, W. Weak and strong convergence theorems for families of nonexpansive mappings and their applications. Annales Universitatis Mariae Curie-Skłodowska. Sectio A. 51 (1997), no. 2, 277-292.

[22] Xu, H. K. Viscosity approximation methods for nonexpansive mappings. J. Math. Anal. Appl. 298 (2004), no. 1, 279-291.

[23] Zhang, Y.; Gui, Y. Strong convergence theorem for split equilibrium problem and fixed point problem in Hilbert spaces. Int. Math. 12 (2017), no. 9, 413-427.

[24] Zhenhua, H. The split equilibrium problem and its convergence algorithms. J. Inequal. Appl. 2012 (2012), no. 1, 1-15.

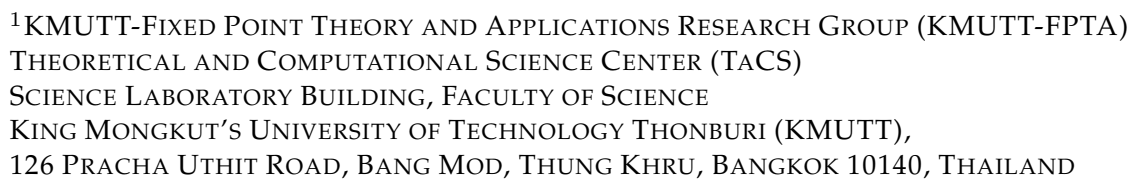

${ }^{2}$ DePARTMENT OF MATHEMATICS

USMANU DANFODIYO UNIVERSITY

P.M.B. 2346, SOKOTO, NigERIA.

Email address: abubakar. jamilu@udusok.edu.ng

${ }^{3}$ FACULTY OF SCIENCE

ENERGY AND ENVIRONMENT

KING MONGKUT'S UNIVERSITY OF TECHNOLOGY NORTH BANGKOK (KMUTNB)

19 MOO 11, NONGLALOK, BANKHAI, RAYONG 21120, THAILAND

Email address: jitsupa.desciee.kmutnb.ac.th 